\title{
Synthesis, characterization, antibacterial and antifungal evaluation of novel cyclohexanone benzoylhydrazones
}

\author{
Gökçe Cihan-Üstündağ' (1), Emel Mataracı-Kara² (D), Gültaze Çapan ${ }^{1}$ (1) \\ 'Department of Pharmaceutical Chemistry, Faculty of Pharmacy, Istanbul University, Istanbul, Turkey \\ 2Department of Pharmaceutical Microbiology, Faculty of Pharmacy, Istanbul University, Istanbul, Turkey
}

ORCID IDs of the authors: G.C.Ü. 0000-0003-0516-6010; E.M.K. 0000-0002-4428-5066; G.Ç. 0000-0002-3026-4563.

Cite this article as: Cihan-Üstündağ G, Mataracı-Kara E, Çapan G (2019). Synthesis, characterization, antibacterial and antifungal evaluation of novel cyclohexanone benzoylhydrazones. Istanbul J Pharm 49 (3): 142-147.

\begin{abstract}
A novel series of benzoyl hydrazones (2a-j) were synthesized and evaluated, in vitro, for antimicrobial activity against selected bacteria and fungi. The structures of the compounds were established by IR, ${ }^{1} \mathrm{H}-\mathrm{NMR},{ }^{13} \mathrm{C}-\mathrm{NMR}$ (APT), electrospray ionization mass spectrometry (ESI-MS) and microanalysis (C, H, N). All of the tested compounds, except for compound $2 \mathrm{~h}$, displayed weak antibacterial properties against Staphylococcus epidermidis ATCC 12228 and Staphylococcus aureus ATCC 29213. Compounds 2a, 2b, 2e, 2f and $2 \mathrm{i}$ further exhibited marginal antifungal activity against Candida parapsilosis.
\end{abstract}

Keywords: Hydrazone, cyclohexanone, antibacterial activity, antifungal activity

\section{INTRODUCTION}

The spread of antibiotic-resistant bacteria is one of the biggest threats to global health. Mortality, morbidity and medical costs due to antibiotic resistance are increasing worldwide. A growing list of infections such as tuberculosis, pneumonia, gonorrhoe, blood poisoning and foodborne diseases, are becoming harder to treat as the antimicrobial agents become less effective (WHO 2018). Every year, around 214,000 deaths in newborns are attributable to drug-resistant pathogens, especially in low- and middleincome countries (Laxminarayan et al. 2016). Despite a growing clinical need, the development of new antibacterial agents to deal with the threat is insufficient. Only two novel antibiotic classes have been discovered in the last 20 years (oxazolidinones and lipopeptides) both of which provide coverage against Gram-positive bacteria (Luepke et al. 2017; Tacconelli et al. 2018). The approval rate of U.S. Food and Drug Administration (FDA) for new antibiotics has fallen to dismally low levels during the past 30 years (Shlaes et al. 2013). There is an urgent need for new antibiotics with activity against resistant microorganisms.

Hydrazide-hydrazones, $\mathrm{R}_{1} \mathrm{R}_{2} \mathrm{C}=\mathrm{N}-\mathrm{NR}_{3} \mathrm{COR}_{4}\left(\mathrm{R}_{1-4}=\right.$ alkyl, aryl or $\left.\mathrm{H}\right)$, are well known as compounds with a wide range of antimicrobial properties (Popiolek 2017). Several $\mathrm{N}$-aroylhydrazones derived from aryl-and heteroaryl hydrazides are emerging in the literature as potential antibacterial agents with wide spectra of activity against both Gram-(+) and Gram-(-) bacteria (Vicini et al. 2002; Gürkök et al. 2009; Moldovan et al. 2011; Xavier et al. 2012; Pieckzonka et al. 2013; Qing Ge et al. 2014; Kaplancıklı et al. 2014; Morjan et al. 2014; Nastasa et al. 2015; Tatar et al. 2016; Sridhar et al. 2016). Some of these derivatives have also been reported to have an inhibitory effect on fungi, especially on Candida species (Vicini et al. 2002; Gürkök et al. 2009; Xavier et al. 2012; Kaplancıklı et al. 2014; Nastasa et al. 2015). In an early report, Backes et al. identified a series of $N^{\prime}$-(2-hydroxybenzylidene) benzohydrazides with potent antifungal activity against two human pathogenic species, Candida albicans and Candida glabrata, at low $\mu$ M concentrations (Backes et al. 2014).

In this study we report the synthesis and structural characterization of novel $\mathrm{N}$-benzoylhydrazones which were obtained by the condensation of 2-hydroxy-4-methoxybenzohydrazide and appropriate cyclohexanone derivatives. These new compounds 
were evaluated for in vitro antibacterial and antifungal activity against a variety of pathogenic bacteria and fungi species.

\section{MATERIALS AND METHODS}

\section{Chemistry}

Melting points were determined in open capillary tubes with a Buchi B-540 melting point apparatus and were uncorrected. Microanalyses were performed on a Thermo Finnigan Flash EA 1112 elemental analyzer. IR spectra were recorded in $\mathrm{KBr}$ discs ( $U_{\text {max }}$ in $\mathrm{cm}^{-1}$ ) on a Shimadzu IRAffinity-1 FTIR spectrophotometer. ${ }^{1} \mathrm{H}-\mathrm{NMR}$ (DMSO- $d_{6}$ ) and ${ }^{13} \mathrm{C}-\mathrm{NMR}$ (APT) (DMSO- $\mathrm{d}_{6}$ ) spectra were run on VarianUNITYINOVA $(500 \mathrm{MHz})$ instrument. Chemical shifts were reported as $\delta$ (ppm) relative to TMS as internal standard and coupling constants ( $($ ) were given in hertz $(\mathrm{Hz})$. MS (ESI+/-) were determined on a Finnigan LCQ Advantage Max mass spectrometer (br: broad/distorted, cyc.: cyclohexylidene, ar:: aromatic).

General procedure for the synthesis of 2-hydroxy-4-methoxy- $N^{\prime}$ [(non)substituted cyclohexylidene]benzohydrazides (2a-j)

A mixture of 2-hydroxy-4-methoxybenzohydrazide (0.003 mol) and an appropriate cyclohexanone $(0.003 \mathrm{~mol})$ in absolute ethanol $(20 \mathrm{~mL})$ was refluxed on a water bath for 5-6 h. After cooling, the product was precipitated by adding distilled water. The solid thus obtained was filtered off and recrystallized from ethanol.

N'-cyclohexylidene-2-hydroxy-4-methoxybenzohydrazide (2a)

Light brown crystals (81\%); mp 200-201 ${ }^{\circ} \mathrm{C} ; \mathrm{IR}(\mathrm{KBr}): \mathrm{U}_{\max } 3311(\mathrm{~N}-$ $\mathrm{H}), 1633$ (C=O), 1612 (C=N), 1587, 1562, 1544, $1519(\mathrm{C}=\mathrm{C}) ;{ }^{1} \mathrm{H}-$ NMR (DMSO- $\left.\mathrm{d}_{6} / 500 \mathrm{MHz}\right): \delta$ 1.59-1.68 (6H, m, CH -cyc.), $2.31-$ $2.35\left(4 \mathrm{H}, \mathrm{m}, \mathrm{CH}\right.$-сус.), $3.77\left(3 \mathrm{H}, \mathrm{s}, 4-\mathrm{OCH}_{3}\right.$-ar. $), 6.46^{2}(1 \mathrm{H}, \mathrm{d}, J=2.5$, H3-ar.), $6.51\left(1 \mathrm{H}^{2}, \mathrm{dd}, J=8.5,2.5, \mathrm{H} 5\right.$-ar. $), 7.86(1 \mathrm{H}, \mathrm{d}, J=8.5, \mathrm{H} 6$-ar.), $10.87(1 \mathrm{H}, \mathrm{s}, \mathrm{NH} / \mathrm{OH}), 12.26(1 \mathrm{H}, \mathrm{s}, \mathrm{NH} / \mathrm{OH}){ }^{13} \mathrm{C}-\mathrm{NMR}$ (APT) (DMSO-d $/ 125$ MHz): $\delta$ 25.48, 26.06, 27.21, 28.13, $35.36\left(\mathrm{CH}_{2}\right.$-cyc.), 55.79 (4-OCH- -ar.), 101.69, 106.61 (C3,C5-ar.), 109.62 (C1-ar.), 131.11 (C6-ar.), 160.50, 163.71, 164.42 (C2,C4-ar.,C=N,C=O). MS (ESI+) m/z (\%): $263.2\left([\mathrm{M}+\mathrm{H}]^{+}, 55.0\right), 285.2\left([\mathrm{M}+\mathrm{Na}]^{+}, 100\right)$. Anal. Calcd for $\mathrm{C}_{14} \mathrm{H}_{18} \mathrm{~N}_{2} \mathrm{O}_{3}$ (262.30): C, 64.10; $\mathrm{H}, 6.92 ; \mathrm{N}, 10.68$. Found: C, 63.74; H, 7.10; N, 10.32.

2-hydroxy-4-methoxy-N'-(4-methylcyclohexylidene)benzohydrazide $(\mathbf{2 b})$

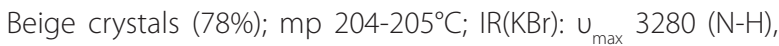
$1651(\mathrm{C}=\mathrm{O}), 1616(\mathrm{C}=\mathrm{N}), 1604,1535,1504(\mathrm{C}=\mathrm{C}) ;{ }^{1} \mathrm{H}-\mathrm{NMR}$ (DMSO- $\left.\mathrm{d}_{6} / 500 \mathrm{MHz}\right): \delta 0.92\left(3 \mathrm{H}, \mathrm{d}, J=6.8,4-\mathrm{CH}_{3}-\mathrm{Cyc}.\right), 1.04-1.20(2 \mathrm{H}$, m, $\mathrm{CH}_{2}$-сус.), 1.67-1.71 (1H, m, CH-cyc.), 1.80-1.87 (2H, m, $\mathrm{CH}_{2}-$ cyc.), $2.01\left(1 \mathrm{H}, \mathrm{td}, J=13.7,5.4, \mathrm{CH}_{2}-\right.$ cyc.), $2.26(1 \mathrm{H}, \mathrm{td}, J=13.2,4.9$, $\mathrm{CH}_{2}$-сус.), $2.42\left(1 \mathrm{H}\right.$, br. d, J=13.7, $\mathrm{CH}_{2}$-cyc.), $2.73(1 \mathrm{H}, \mathrm{br} . \mathrm{d}, J=14.1$, $\mathrm{CH}_{2}$-сус.), 3.77 (3H, s, 4-OCH- -ar.), $6.46(1 \mathrm{H}, \mathrm{d}, J=2.4, \mathrm{H} 3$-ar.), $6.51(1 \mathrm{H}, \mathrm{dd}, J=8.8,2.4, \mathrm{H} 5$-ar.), $7.86(1 \mathrm{H}, \mathrm{d}, J=8.8, \mathrm{H} 6$-ar. $), 10.86$ $(1 \mathrm{H}, \mathrm{s}, \mathrm{NH} / \mathrm{OH}), 12.27(1 \mathrm{H}, \mathrm{s}, \mathrm{NH} / \mathrm{OH}) ;{ }^{13} \mathrm{C}-\mathrm{NMR}$ (APT) (DMSO$\left.\mathrm{d}_{6} / 125 \mathrm{MHz}\right): \delta 21.73\left(4-\mathrm{CH}_{3}-\right.$-сyc.), $27.27\left(\mathrm{CH}_{2}-\mathrm{cyc}.\right), 31.49(\mathrm{CH}-$ cyc.), 33.97, 34.57, $35.16\left(\mathrm{CH}_{2}\right.$-сус.), $55.80\left(4-\mathrm{OCH}_{3}\right.$-ar. $), 101.69$, 106.58 (C3,C5-ar.), 109.60 (C1-ar.), 131.09 (C6-ar.), 160.58, 163.72, 164.02, 164.42 (C2,C4-ar.,C=N,C=O). MS (ESI+) m/z (\%): 277.1 $\left([\mathrm{M}+\mathrm{H}]^{+}, 100\right)$. Anal. Calcd for $\mathrm{C}_{15} \mathrm{H}_{20} \mathrm{~N}_{2} \mathrm{O}_{3}$ (276.33): $\mathrm{C}, 65.20 ; \mathrm{H}$, 7.30; N, 10.14. Found: C, 65.03; H, 7.50; N, 9.88.
N'-(4-ethylcyclohexylidene)-2-hydroxy-4-methoxybenzohydrazide (2c)

White crystals (86\%); mp 166-168 ${ }^{\circ} \mathrm{C} ; \mathrm{IR}(\mathrm{KBr}): \mathrm{U}_{\max } 3305(\mathrm{~N}-\mathrm{H}), 1633$ $(\mathrm{C}=\mathrm{O}), 1610(\mathrm{C}=\mathrm{N}), 1580,1550,1516(\mathrm{C}=\mathrm{C}) ;{ }^{1} \mathrm{H}-\mathrm{NMR}$ (DMSO$\left.\mathrm{d}_{6} / 500 \mathrm{MHz}\right): \delta 0.89\left(3 \mathrm{H}, \mathrm{t}, \mathrm{J}=7.3,4-\mathrm{CH}_{2} \mathrm{CH}_{3}\right.$-Cyc.), 1.06-1.18 (2H, m, $\mathrm{CH}_{2}$-сус.), 1.25 (2H, quin., J=7.3, 4- $\mathrm{CH}_{2} \mathrm{CH}_{3}$-cyc.), 1.44-1.48 (1H, m, CH-cyc.), 1.86-2.03 (3H, m, $\mathrm{CH}_{2}$-cyc.), $2.25\left(1 \mathrm{H}, \mathrm{td}, J=13.5,4.9, \mathrm{CH}_{2}-\right.$ cyc.), $2.43\left(1 \mathrm{H}, \mathrm{br} . \mathrm{d}, J=13.7, \mathrm{CH}_{2}-\mathrm{cyc}.\right), 2.73\left(1 \mathrm{H}, \mathrm{br} . \mathrm{d}, J=14.2, \mathrm{CH}_{2}-\right.$ cyc.), 3.77 (3H, s, 4-OCH -ar.), $6.46(1 \mathrm{H}, \mathrm{d}, J=2.5, \mathrm{H} 3$-ar.), $6.51(1 \mathrm{H}$, $\mathrm{dd}, J=9.0,2.5, \mathrm{H} 5$-ar.), 7.86 (1H, d, J=8.5, H6-ar.), $10.86(1 \mathrm{H}, \mathrm{s}, \mathrm{NH} /$ $\mathrm{OH}), 12.26(1 \mathrm{H}, \mathrm{s}, \mathrm{NH} / \mathrm{OH}) ;{ }^{13} \mathrm{C}-\mathrm{NMR}$ (APT) (DMSO-d $\left./ 125 \mathrm{MHz}\right): \delta$ $11.95\left(4-\mathrm{CH}_{2} \mathrm{CH}_{3}-\right.$-сус.), 27.25, 28.63, 31.64, 32.72, $34.52\left(\mathrm{CH}_{2}\right.$-cyc., 4- $\mathrm{CH}_{2} \mathrm{CH}_{3}$-сус.), 38.06 (CH-cyc.), 55.79 (4-OCH${ }_{3}$-ar.), 101.69, 106.60 (C3,C5-ar.), 109.60 (C1-ar.), 131.10 (C6-ar.), 160.53, 163.71, 164.03, 164.72 (C2,C4-ar., $C=N, C=O)$. Anal. Calcd for $\mathrm{C}_{16} \mathrm{H}_{22} \mathrm{~N}_{2} \mathrm{O}_{3}$ (290.36): C, 66.18; H, 7.64; N, 9.65. Found: C, 66.40; H, 7.80; N, 9.52.

2-hydroxy-4-methoxy-N'-(4-propylcyclohexylidene)benzohydrazide (2d)

White crystals (87\%); mp $158-160^{\circ} \mathrm{C} ; \mathrm{IR}(\mathrm{KBr}): \mathrm{U}_{\max } 3304(\mathrm{~N}-\mathrm{H})$, $1637(\mathrm{C}=\mathrm{O}), 1612(\mathrm{C}=\mathrm{N}), 1558,1519(\mathrm{C}=\mathrm{C})$; ${ }^{1} \mathrm{H}-\mathrm{NMR}$ (DMSO$\left.\mathrm{d}_{6} / 500 \mathrm{MHz}\right): \delta 0.87\left(3 \mathrm{H}, \mathrm{t}, J=7.3,4-\mathrm{CH}_{2} \mathrm{CH}_{2} \mathrm{CH}_{3}-\mathrm{cyc}.\right), 1.07-1.22$ (4H, m, 4- $\left.\mathrm{CH}_{2} \mathrm{CH}_{2} \mathrm{CH}_{3}, \mathrm{CH}_{2}-\mathrm{Cyc}.\right), 1.28-1.34\left(2 \mathrm{H}, \mathrm{m}, 4-\mathrm{CH}_{2} \mathrm{CH}_{2} \mathrm{CH}_{3}-\right.$ сус.), 1.54-1.58 (1H, m, CH-cyc.), 1.85-2.02 (3H, m, $\mathrm{CH}_{2}-$ cyc.), 2.25 $\left(1 \mathrm{H}, \mathrm{td}, J=13.8,4.9, \mathrm{CH}_{2}\right.$-сус.), $2.43\left(1 \mathrm{H}, \mathrm{br} . \mathrm{d}, J=14.2, \mathrm{CH}_{2}-\right.$ cyc.), $2.73\left(1 \mathrm{H}\right.$, br. d, $J=13.7, \mathrm{CH}_{2}$-cyc.), $3.77\left(3 \mathrm{H}, \mathrm{s}, 4-\mathrm{OCH}_{3}\right.$-ar.), 6.46 $(1 \mathrm{H}, \mathrm{d}, J=2.5, \mathrm{H} 3$-ar. $), 6.51(1 \mathrm{H}, \mathrm{dd}, J=8.8,2.5, \mathrm{H} 5$-ar. $), 7.86(1 \mathrm{H}, \mathrm{d}$, $J=8.5, \mathrm{H} 6$-ar.), $10.86(1 \mathrm{H}, \mathrm{s}, \mathrm{NH} / \mathrm{OH}), 12.26(1 \mathrm{H}, \mathrm{s}, \mathrm{NH} / \mathrm{OH}) ;{ }^{13} \mathrm{C}-$ NMR (APT) (DMSO-d $/ 125 \mathrm{MHz}): \delta 14.65$ (4- $\mathrm{CH}_{2} \mathrm{CH}_{2} \mathrm{CH}_{3}$-Cyc.), $20.12,27.28,32.02,33.10,34.54,38.25\left(\mathrm{CH}_{2}-\mathrm{Cyc} ., 4-\mathrm{CH}_{2} \mathrm{CH}_{2} \mathrm{CH}_{3}-\right.$ сус.), 36.04 (CH-сус.), 55.78 (4- $\mathrm{OCH}_{3}$-ar.), 101.69, 106.59 (C3,C5ar.), 109.60 (C1-ar.), 131.09 (C6-ar.), 160.55, 163.71, 163.99, 164.71 (C2,C4-ar., $\mathrm{C}=\mathrm{N}, \mathrm{C}=\mathrm{O})$. Anal. Calcd for $\mathrm{C}_{17} \mathrm{H}_{24} \mathrm{~N}_{2} \mathrm{O}_{3}$ (304.38): C, 67.08; H, 7.95; N, 9.20. Found: C, 67.33; H, 8.11; N, 9.13.

N'-(4-tert-butylcyclohexylidene)-2-hydroxy-4-methoxybenzohydrazide (2e)

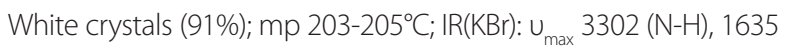
$(\mathrm{C}=\mathrm{O}), 1609(\mathrm{C}=\mathrm{N}), 1558,1516,1480(\mathrm{C}=\mathrm{C}) ;{ }^{1} \mathrm{H}-\mathrm{NMR}$ (DMSO$\left.\mathrm{d}_{6} / 500 \mathrm{MHz}\right): \delta 0.86\left(9 \mathrm{H}, \mathrm{s}, 4-\mathrm{C}\left(\mathrm{CH}_{3}\right)_{3}-\mathrm{Cyc}.\right), 1.13-1.24\left(2 \mathrm{H}, \mathrm{m}, \mathrm{CH}_{2}-\right.$ сус.), 1.31-1.36 (1H, m, CH-cyc.), 1.88-1.97 (3H, m, $\left.\mathrm{CH}_{2}-\mathrm{cyc}.\right), 2.25$ $\left(1 \mathrm{H}, \mathrm{td}, J=13.2,4.9, \mathrm{CH}_{2}-\right.$ cyc.) $2.46\left(1 \mathrm{H}, \mathrm{br} . \mathrm{d}, J=13.7, \mathrm{CH}_{2}\right.$-сус.), $2.81\left(1 \mathrm{H}, \mathrm{br} . \mathrm{d}, J=14.2, \mathrm{CH}_{2}\right.$-cyc.), $3.77\left(3 \mathrm{H}, \mathrm{s}, 4-\mathrm{OCH}_{3}\right.$-ar.), $6.46(1 \mathrm{H}$, $\mathrm{d}, J=2.5, \mathrm{H3}$-ar.), 6.51 (1H, dd, J=9.0, 2.5, H5-ar.), $7.86(1 \mathrm{H}, \mathrm{d}, J=8.5$, H6-ar.), 10.85 (1H, s, NH/OH), $12.27(1 \mathrm{H}, \mathrm{s}, \mathrm{NH} / \mathrm{OH}){ }_{i}^{13} \mathrm{C}-\mathrm{NMR}$ (APT) (DMSO-d $/ 125 \mathrm{MHz}): \delta 26.75,27.25\left(\mathrm{CH}_{2}-\mathrm{Cyc}.\right), 27.82\left(4-\mathrm{C}_{6} \mathrm{CH}_{3}\right)_{3}{ }^{-}$ cyc.), 32.62 (4- $\underline{\mathrm{C}}\left(\mathrm{CH}_{3}\right)_{3}$-сус.), $34.98\left(\mathrm{CH}_{2}-\right.$-сyc.), 46.80 (CH-cyc.), 55.78 (4-OCH -ar.), 101.69, 106.60 (C3,C5-ar.), 109.58 (C1-ar.), 131.07 (C6ar.), 160.51, 163.71, 164.05, 164.67 (C2,C4-ar., $C=N, C=O)$. MS (ESI+) $\mathrm{m} / \mathrm{z}(\%): 319.2\left([\mathrm{M}+\mathrm{H}]^{+}, 100\right)$. Anal. Calcd for $\mathrm{C}_{18} \mathrm{H}_{26} \mathrm{~N}_{2} \mathrm{O}_{3}$ (318.41): $\mathrm{C}$, $67.90 ; \mathrm{H}, 8.23 ; \mathrm{N}, 8.80$. Found: $\mathrm{C}, 67.53 ; \mathrm{H}, 8.25 ; \mathrm{N}, 8.83$.

2-hydroxy-4-methoxy-N'-(3-methylcyclohexylidene)benzohydrazide (2f)

White crystals (95\%); mp $167-169^{\circ} \mathrm{C} ; \mathrm{IR}(\mathrm{KBr}): \mathrm{U}_{\max } 3311(\mathrm{~N}-\mathrm{H})$, $1622(\mathrm{C}=\mathrm{O}), 1613(\mathrm{C}=\mathrm{N}), 1581,1545,1508(\mathrm{C}=\mathrm{C}) ;{ }^{1} \mathrm{H}-\mathrm{NMR}(\mathrm{DM}-$ 
SO-d 6 /500 MHz): $\delta$ 0.94, $0.95\left(3 \mathrm{H}, 2 \mathrm{~d}, J=6.3,3-\mathrm{CH}_{3}-\right.$ cyc.), 1.12$1.20\left(1 \mathrm{H}, \mathrm{m}, \mathrm{CH} / \mathrm{CH}_{2}-\mathrm{Cyc}.\right), 1.37-1.49\left(1 \mathrm{H}, \mathrm{m}, \mathrm{CH} / \mathrm{CH}_{2}-\mathrm{cyc}.\right), 1.60-$ $1.94\left(4 \mathrm{H}, \mathrm{m}, \mathrm{CH} / \mathrm{CH}_{2}-\mathrm{cyc}.\right), 2.17\left(1 \mathrm{H}, \mathrm{td}, J=13.5,5.4, \mathrm{CH}_{2}-\mathrm{cyc}.\right)$, 2.35-2.44 (1 H, m, $\mathrm{CH}_{2}$-сус.), 2.65-2.71 (1 H, m, $\mathrm{CH}_{2}$-cyc.), $3.77(3 \mathrm{H}$, $\mathrm{s}, 4-\mathrm{OCH}_{3}$-ar.), 6.46, 6.47 (1H, 2d, J=2.5, H3-ar.), 6.50-6.52 (1H, m, H5-ar.), 7.87, 7.89 (1H, 2d, J=8.8, H6-ar.), 10.89 (1H, s, NH/OH), $12.27(1 \mathrm{H}, \mathrm{s}, \mathrm{NH} / \mathrm{OH}) ;{ }^{13} \mathrm{C}-\mathrm{NMR}$ (APT) (DMSO-d $\left./ 125 \mathrm{MHz}\right): \delta$ 22.17, 22.35 (3- $\mathrm{CH}_{3}$-сус.), 24.81, 25.91, $27.51\left(\mathrm{CH}_{2}-\mathrm{Cyc}\right.$ ), 32.77, 33.70 (CH-cyc.), 33.79, 34.83, 36.00, $43.31\left(\mathrm{CH}_{2}-\mathrm{Cyc}.\right), 55.78$ (4-OCH 3 -ar.), 101.69, 106.58 (C3,C5-ar.), 109.58, 109.68 (C1-ar.), 131.09, 131.18 (C6-ar.), 160.50, 160.60, 163.72, 163.91, 164.33 (C2,C4-ar.,C=N,C=O). ). MS (ESI-) m/z (\%): 275.5 ([M-H]; 100). Anal. Calcd for $\mathrm{C}_{15} \mathrm{H}_{20} \mathrm{~N}_{2} \mathrm{O}_{3}$ (276.33): C, 65.20; $\mathrm{H}, 7.30 ; \mathrm{N}, 10.14$. Found: $\mathrm{C}, 65.30 ; \mathrm{H}, 7.52 ; \mathrm{N}, 10.06$.

2-hydroxy-4-methoxy-N'-(3,3,5-trimethylcyclohexylidene)benzohydrazide (2g)

White crystals (94\%); mp 203-206 ${ }^{\circ} \mathrm{C} ; \mathrm{IR}(\mathrm{KBr}): \mathrm{U}_{\max } 3304(\mathrm{~N}-\mathrm{H})$, $1658(\mathrm{C}=\mathrm{O}), 1627(\mathrm{C}=\mathrm{N}), 1604,1543,1504(\mathrm{C}=\mathrm{C}){ }^{\prime}{ }^{1} \mathrm{H}-\mathrm{NMR}(\mathrm{DM}-$ SO-d 6 /500 MHz): $\delta 0.78,0.83\left(3 \mathrm{H}, 2 \mathrm{~s}, 3-\mathrm{CH}_{3}-\mathrm{Cyc}\right.$ ), 0.93-0.95 (3H, m, 5- $\mathrm{CH}_{3}$-сус.), 0.99, $1.02\left(3 \mathrm{H}, 2 \mathrm{~s}, 3-\mathrm{CH}_{3}\right.$-сус.), 1.09-1.15 (1H, m, $\mathrm{CH} / \mathrm{CH}_{2}$-сус.), 1.44, $1.48\left(1 \mathrm{H}\right.$, br. $\left.2 \mathrm{~d}, \mathrm{~J}=13.1, \mathrm{CH} / \mathrm{CH}_{2}-\mathrm{cyc}.\right), 1.72-$ $1.81\left(2 \mathrm{H}, \mathrm{m}, \mathrm{CH} / \mathrm{CH}_{2}\right.$-сус.), 2.03-2.12 (1H, m, CH/CH - -cyc.), 2.38 $\left(1 \mathrm{H}\right.$, br. d, J=9.8, $\mathrm{CH} / \mathrm{CH}_{2}$-cyc.), $2.74\left(1 \mathrm{H}\right.$, br. $\mathrm{d}, J=13.2, \mathrm{CH}_{2}$-сус.), 3.76, $3.77\left(3 \mathrm{H}, 2 \mathrm{~s}, 4-\mathrm{OCH}_{3}\right.$-ar. $), 6.46,6.47(1 \mathrm{H}, 2 \mathrm{~d}, J=2.5, \mathrm{H} 3-$ ar.), 6.50-6.54 (1H, m, H5-ar.), 7.87, $7.89(1 \mathrm{H}, 2 \mathrm{~d}, \mathrm{~J}=9.0, \mathrm{H} 6$-ar.), $10.92(1 \mathrm{H}, \mathrm{s}, \mathrm{NH} / \mathrm{OH}), 12.11,12.32(1 \mathrm{H}, 2 \mathrm{~s}, \mathrm{NH} / \mathrm{OH}) ;{ }^{13} \mathrm{C}-\mathrm{NMR}$ (APT) (DMSO-d $/ 125 \mathrm{MHz}): \delta 22.57,22.74,25.62,25.65,28.44$ (3- $\left(\mathrm{CH}_{3}\right)_{2}, 5-\mathrm{CH}_{3}$-Cyc.), 30.00, 32.18 ( $\mathrm{CH}$-сyc.), 34.00, 34.61, 35.65 ( $\mathrm{CH}_{2}$-сyc.), 43.20, 47.57, $47.66\left(\mathrm{CH}_{2}\right.$-сyc., C3-cyc.), $55.80\left(4-\mathrm{OCH}_{3}-\right.$ ar.), 101.71, 101.77, 106.57, 106.64 (C3,C5-ar.), 109.48, 110.32 (C1-ar.), 131.00, 131.68 (C6-ar.), 159.61, 160.74, 161.95, 163.62, 163.74, 164.10, 164.31 (C2,C4-ar., $C=N, C=O)$. MS (ESI-) m/z (\%): 303.6 ([M-H] ; , 100). Anal. Calcd for $\mathrm{C}_{17} \mathrm{H}_{24} \mathrm{~N}_{2} \mathrm{O}_{3}$ (304.38): C, 67.08; H, 7.95; N, 9.20. Found: C, 67.36; H, 8.12; N, 9.08.

N'-[4-(acetylamino)cyclohexylidene]-2-hydroxy-4-methoxybenzohydrazide $\mathbf{( 2 h )}$

White flakes (90\%); mp 242-245ㄷ $\mathrm{C} I \mathrm{R}(\mathrm{KBr}): \mathrm{U}_{\max } 3342,3298(\mathrm{~N}-\mathrm{H})$, 1631 (C=O), 1609 (C=N), 1537, 1514, 1497 (C=C); ${ }^{1} \mathrm{H}-\mathrm{NMR}$ (DMSO$\left.\mathrm{d}_{6} / 500 \mathrm{MHz}\right): \delta 1.36-1.50\left(2 \mathrm{H}, \mathrm{m}, \mathrm{CH}_{2}-\mathrm{Cyc}.\right), 1.81\left(3 \mathrm{H}, \mathrm{s}, 4-\mathrm{NHCOCH}_{3}-\right.$ сус.), 1.87-1.94 (2H, m, $\mathrm{CH}_{2}$-сус.), 2.13-2.19 (1 $1 \mathrm{H}, \mathrm{m}, \mathrm{CH}_{2}-$-сус.), 2.35 $\left(1 \mathrm{H}, \mathrm{td}, J=13.3,4.9, \mathrm{CH}_{2}\right.$-сус.), 2.44-2.49 $\left(1 \mathrm{H}, \mathrm{m}, \mathrm{CH}_{2}-\mathrm{cyc}.\right), 2.68(1 \mathrm{H}$, br. d, J=15.1, $\mathrm{CH}_{2}$-cyc.), $3.77\left(3 \mathrm{H}, \mathrm{s}, 4-\mathrm{OCH}_{3}\right.$-ar.), 3.82-3.90 (1H, m, (H-cyc.), 6.46 (1H, d, J=2.5, H3-ar.), $6.51(1 \mathrm{H}, \mathrm{dd}, J=8.8,2.5, \mathrm{H} 5$-ar.), $7.86(1 \mathrm{H}, \mathrm{d}, J=7.5, \mathrm{H} 6-\mathrm{ar}),. 10.88(1 \mathrm{H}, \mathrm{s}, \mathrm{NH} / \mathrm{OH}), 12.26(1 \mathrm{H}, \mathrm{s}, \mathrm{NH} /$ $\mathrm{OH}) ;{ }^{13} \mathrm{C}-\mathrm{NMR}$ (APT) (DMSO-d $\left./ 125 \mathrm{MHz}\right): \delta 23.20\left(4-\mathrm{NHCOCH}_{3}-\right.$ сус.), 25.71, 31.18, 32.35, 32.96 ( $\mathrm{CH}_{2}-$-сус.), 46.40 (CH-cyc.), 55.80 (4-OCH -ar.), 101.69, 106.64 (C3,C5-ar.), 109.50 (C1-ar.), 131.10 (C6ar.), 160.63, 163.24, 163.78, 164.11 (C2,C4-ar., $C=N, C=O), 168.92$ (4- $\mathrm{NHCOCH}_{3}$-cyc.). Anal. Calcd for $\mathrm{C}_{16} \mathrm{H}_{21} \mathrm{~N}_{3} \mathrm{O}_{4}$ (319.36): $\mathrm{C}, 60.17 ; \mathrm{H}$, 6.63; N, 13.16. Found: C, 59.73; H, 7.05; N, 12.93.

N'-[2-(2-cyanoethyl)cyclohexylidene]-2-hydroxy-4-methoxybenzohydrazide (2i)

White crystals (69\%); mp 147-149 ${ }^{\circ} \mathrm{C} ; \mathrm{IR}(\mathrm{KBr}): \mathrm{U}_{\max } 3304(\mathrm{~N}-\mathrm{H})$, $1649(\mathrm{C}=\mathrm{O}), 1614(\mathrm{C}=\mathrm{N}), 1543,1508(\mathrm{C}=\mathrm{C})$; ${ }^{1} \mathrm{H}-\mathrm{NMR}$ (DMSO- $\left.\mathrm{d}_{6} / 500 \mathrm{MHz}\right): \delta$ 1.32-1.40 (1H, m, CH/CH - sp.), 1.49-1.74 (5H, m, 2- $\mathrm{CH}_{2} \mathrm{CH}_{2} \mathrm{CN}$-cyc. and/or $\mathrm{CH} / \mathrm{CH}_{2}$-sp.), 1.88-1.94 (1H, m, $\mathrm{CH} / \mathrm{CH}_{2}-$ sp.), 2.04-2.11 (2H, m, 2- $\underline{\mathrm{H}}_{2} \mathrm{CH}_{2} \mathrm{CN}-\mathrm{cyc}$. or $\mathrm{CH} / \mathrm{CH}_{2}$-sp.), 2.37-2.41 $\left(1 \mathrm{H}, \mathrm{m}, \mathrm{CH} / \mathrm{CH}_{2}-\mathrm{sp}.\right), 2.60-2.65\left(3 \mathrm{H}, \mathrm{m}, 2-\mathrm{CH}_{2} \mathrm{CH}_{2} \mathrm{CN}-\mathrm{cyc} ., \mathrm{CH} /\right.$ $\mathrm{CH}_{2}$-sp.), 3.77 (3H, s, 4-OCH -ar.), $6.47(1 \mathrm{H}, \mathrm{d}, J=2.5, \mathrm{H} 3$-ar.), 6.52 $(1 \mathrm{H}, \mathrm{dd}, J=8.5,2.5, \mathrm{H} 5$-ar. $), 7.87(1 \mathrm{H}, \mathrm{d}, J=8.5, \mathrm{H} 6$-ar. $), 10.94(1 \mathrm{H}$, $\mathrm{s}, \mathrm{NH} / \mathrm{OH}), 12.22(1 \mathrm{H}, \mathrm{s}, \mathrm{NH} / \mathrm{OH}) ;{ }^{13} \mathrm{C}-\mathrm{NMR}$ (APT) (DMSO-d/125 $\mathrm{MHz}): \delta 14.78$ (2- $\mathrm{CH}_{2} \mathrm{CH}_{2} \mathrm{CN}-\mathrm{cyc}$.), 24.04, 26.51, $27.45\left(\mathrm{CH}_{2}-\mathrm{cyc}\right.$., $\left.2-\mathrm{CH}_{2} \mathrm{CH}_{2} \mathrm{CN}-\mathrm{cyc}\right), 33.30,41.88\left(\mathrm{CH}_{2}\right.$-сyc.), 43.20 (CH-cyc.), 55.80 (4-OCH ${ }_{3}$-ar.), 101.71, 106.66 (C3,C5-ar.), 109.58 (C1-ar.), 121.31 (2- $\mathrm{CH}_{2} \mathrm{CH}_{2}$ ( $\left.\mathrm{N}-\mathrm{Cyc}\right), 131.12$ (C6-ar.), 160.57, 163.77, 164.10, 165.46 (C2,C4-ar., $\mathrm{C}=\mathrm{N}, \mathrm{C}=\mathrm{O})$. Anal. Calcd for $\mathrm{C}_{17} \mathrm{H}_{21} \mathrm{~N}_{3} \mathrm{O}_{3}$ (315.37): $\mathrm{C}, 64.74$; $H, 6.71 ; \mathrm{N}, 13.32$. Found: C, 64.29; H, 6.86; N, 13.46.

2-hydroxy-4-methoxy-N'-(4-phenylcyclohexylidene) benzohydrazide (2j)

White crystals (84\%); mp 221-222 ${ }^{\circ} \mathrm{C} ; \mathrm{IR}(\mathrm{KBr}): \mathrm{U}_{\max } 3263(\mathrm{~N}-\mathrm{H})$, $1638(\mathrm{C}=\mathrm{O}) 1607(\mathrm{C}=\mathrm{N}), 1543,1502(\mathrm{C}=\mathrm{C})$; ${ }^{1} \mathrm{H}-\mathrm{NM} \mathrm{R}$ (DMSO$\left.\mathrm{d}_{6} / 500 \mathrm{MHz}\right): \delta 1.60-1.75\left(2 \mathrm{H}, \mathrm{m}, \mathrm{CH}_{2}-\mathrm{cyc}.\right), 1.97-2.04\left(2 \mathrm{H}, \mathrm{m}, \mathrm{CH}_{2}-\right.$ сус.), $2.16\left(1 \mathrm{H}, \mathrm{td}, J=14.1,5.4, \mathrm{CH}_{2}-\right.$ сус. $), 2.46(1 \mathrm{H}, \mathrm{td}, J=13.7,4.9$, $\mathrm{CH}_{2}$-сус.), $2.56\left(1 \mathrm{H}, \mathrm{br} . \mathrm{d}, \mathrm{J}=13.7, \mathrm{CH}_{2}-\mathrm{cyc}.\right), 2.85-2.91(2 \mathrm{H}, \mathrm{m}, \mathrm{CH}-$ cyc., $\mathrm{CH}_{2}$-cyc.), 3.77 (3H, s, 4-OCH - -ar.), $6.47(1 \mathrm{H}, \mathrm{d}, J=2.5, \mathrm{H} 3$-ar.), $6.52(1 \mathrm{H}, \mathrm{dd}, J=8.5,2.5, \mathrm{H} 5$-ar. $), 7.18-7.31\left(5 \mathrm{H}, \mathrm{m}, 4-\mathrm{C}_{6} \mathrm{H}_{5}\right.$-cyc.), 7.88 $(1 \mathrm{H}, \mathrm{d}, J=9.0, \mathrm{H} 6$-ar.), $10.94(1 \mathrm{H}, \mathrm{s}, \mathrm{NH} / \mathrm{OH}), 12.27(1 \mathrm{H}, \mathrm{s}, \mathrm{NH} / \mathrm{OH})$; ${ }^{13} \mathrm{C}-\mathrm{NMR}$ (APT) (DMSO-d $\left./ 125 \mathrm{MHz}\right): \delta 27.81,33.24,34.27,35.13$ $\left(\mathrm{CH}_{2}\right.$-сус.), 42.93 (CH-cyc.), 55.80 (4-OCH- -ar.), 101.71, 106.65 (C3,C5-ar.), 109.62 (C1-ar.), 126.61, 127.16, $128.84\left(4-\mathrm{C}_{6} \mathrm{H}_{5}(\mathrm{C} 2-\right.$ 6)-сус.), 131.17 (C6-ar.), 146.19 (4- $\mathrm{C}_{6} \mathrm{H}_{5}(\mathrm{C} 1)$-cyc.), 160.55, 163.46, 163.75, 164.04 ( $\mathrm{C} 2, \mathrm{C} 4$-ar., $\mathrm{C}=\mathrm{N}, \mathrm{C}=\mathrm{O})$. Anal. Calcd for $\mathrm{C}_{20} \mathrm{H}_{22} \mathrm{~N}_{2} \mathrm{O}_{3}$ (338.40): C, 70.99; H, 6.55; N, 8.28. Found: C, 70.57; H, 6.67; N, 8.36.

\section{Antibacterial and Antifungal Activity Assays}

The in vitro antimicrobial activity of compounds $\mathbf{2 a - j}$ was evaluated against four Gram negatif bacteria (Pseudomonas aeruginosa ATCC 27853, Escherichia coli ATCC 25922, Klebsiella pneumoniae ATCC 4352, Proteus mirabilis ATCC 14153), three Gram positive bacteria (Staphylococcus aureus ATCC 29213, Staphylococcus epidermidis ATCC 12228, Enterococcus faecalis ATCC 29212) and three fungi (Candida albicans ATCC 10231, Candida parapsilosis ATCC 22019 and Candida tropicalis ATCC 750) using the microbroth dilution method according to the Clinical Laboratory Standards Institute (CLSI) recommendations (CLSI 1997; CLSI 2006) and compared with the standard drugs.

Serial twofold dilutions ranging from $2500 \mu \mathrm{g} / \mathrm{mL}$ to $1.22 \mu \mathrm{g} /$ $\mathrm{mL}$ were prepared in the test medium, i.e. Mueller-Hinton broth for bacteria and RPMI-1640 medium for yeast strains. The inoculum was prepared using a 4-6 h broth culture of each bacteria type and $24 \mathrm{~h}$ culture of yeast strains adjusted to a turbidity equivalent to $0.5 \mathrm{McF}$ Frland standard, diluted in broth media to give a final concentration in the test tray of $5 \times 10^{5}$ $\mathrm{cfu} / \mathrm{mL}$ for bacteria and $5 \times 10^{3} \mathrm{cfu} / \mathrm{mL}$ for yeast. The trays were covered and placed into plastic bags to prevent evaporation. The bacteria trays were incubated at $35^{\circ} \mathrm{C}$ for $18-20 \mathrm{~h}$ while the yeast-containing trays were incubated at $35^{\circ} \mathrm{C}$ for $46-50 \mathrm{~h}$. The MIC was defined as the lowest concentration of compound giving complete inhibition of visible growth. As a control, antimicrobial effects of the solvents against the tested microorganisms were also investigated. 


\section{RESULTS AND DISCUSSION}

\section{Chemistry and Structural Characterization}

The synthetic pathway for the preparation of the target hydrazones (2a-j) are illustrated in Scheme 1. Reactions occured readily under mild temperatures. The structures of the obtained compounds were established using IR, ${ }^{1} \mathrm{H}-\mathrm{NMR},{ }^{13} \mathrm{C}-$ NMR (APT), electrospray ionization mass spectrometry (ESIMS) and microanalytical data.

\section{Scheme 1}

IR spectra of the new hydrazone derivatives $\mathbf{2} \mathbf{a}$-j showed single $\mathrm{N}-\mathrm{H}$ band in the $3263-3311 \mathrm{~cm}^{-1}$ region, while the IR spectrum of the starting hydrazide (1) exhibited three seperate $\mathrm{N}-\mathrm{H}$ strechings at 3149,3275 and $3319 \mathrm{~cm}^{-1}$. The $\mathrm{C}=\mathrm{O}$ groups of compounds $\mathbf{1}$ and $\mathbf{2 a - j}$ absorbed in the 1635 and 1622-1658 $\mathrm{cm}^{-1}$ regions, respectively. No phenolic $\mathrm{O}-\mathrm{H}$ streching vibrations were observed in the IR spectra of the hydrazide (1) or hydrazones $(\mathbf{2} \mathbf{a}-\mathbf{j})$. Absence of the $\mathrm{O}-\mathrm{H}$ bands in the expected regions is presumably due to the strong intramolecular hydrogen bonding between the phenolic $\mathrm{O}-\mathrm{H}$ and $\mathrm{C}=\mathrm{O}$ groups (Silverstein et al. 2005).

${ }^{1} \mathrm{H}-\mathrm{NMR}$ spectra displayed the $\mathrm{N}-\mathrm{H}$ and $\mathrm{O}-\mathrm{H}$ resonances in the $\delta$ 10.85-10.94 ppm and $\delta$ 12.11-12.32 ppm regions as singlets. The resonances of the $-\mathrm{OCH}_{3}$ group and aromatic hydrogens were observed in the $\delta$ 3.76-3.77 ppm and $\delta$ 6.46-7.89 ppm, respectively. The splitting patterns of the aromatic $\mathrm{H} 3, \mathrm{H} 5, \mathrm{H} 6$ hydrogens were in accordance with the 1,2,4-trisubstituted aromatic ring system. The aliphatic protons of the cyclohexane residue resonated at about $\delta$ 1.04-3.79 ppm region depending on the substituents on the ring system.

Carbon assignments were made on the basis of APT experiments which provided information about the number of protons attached to a ${ }^{13} \mathrm{C}$ atom. The quaternary $\mathrm{C}=\mathrm{N}$ and $\mathrm{C}=\mathrm{O}$ carbon resonances appeared as positive signals in downfield region together with the aromatic quaternary C2 and C4 carbon resonances ( $\delta$ 159.61-165.46 ppm). Observation of upfield resonances ( $\delta$ 11.95-47.66 ppm) assigned to the $\mathrm{CH} / \mathrm{CH}_{2}$ carbons of the cyclohexane residue further proved the intended conversion.

The proton spectra of compounds $\mathbf{2} \mathbf{f}$ and $\mathbf{2 g}$ displayed two sets of signals for some protons. Aromatic protons of $\mathbf{2} \mathbf{f}$ and $\mathbf{2 g}$ absorbed as two doublets ( $\mathrm{H} 3$ and $\mathrm{H} 6$ ) and distorted multiplets (H5). Methyl substituents on the cyclohexane ring appeared as duplicated doublets for both compounds. The $\mathrm{OH} /$ $\mathrm{CONH}$ resonance about $12.00 \mathrm{ppm}$ and the $-\mathrm{OCH}_{3}$ resonance of compound $\mathbf{2} \mathbf{g}$ were also detected as two separate singlets. Similarly, two signal sets appeared for most of the carbon atoms in the APT spectra of compounds $\mathbf{2} \mathbf{f}$ and $\mathbf{2 g}$. The multiplicity in the signals pointed to the presence of two isomers due to the restricted rotation about the $\mathrm{N}=\mathrm{C}$ double bond. It is assumed that the methyl substituents at 3-or 5-positions interrupt the symmetry of the molecules and give rise to the formation of $E$ and $Z$ isomers for compounds $\mathbf{2 f}$ and $\mathbf{2 g}$.

ESI-MS was used to verify the molecular weights of compounds $\mathbf{2 a}, \mathbf{2 b}, \mathbf{2 e}, \mathbf{2 f}$ and $\mathbf{2 g}$. Compounds $\mathbf{2 a}, \mathbf{2} \mathbf{b}$ and $\mathbf{2 e}$ were analyzed under negative-ion ESI conditions while compounds $\mathbf{2 f}$ and $\mathbf{2 g}$ were analyzed under positive-ion ESI conditions. Deprotonated $[\mathrm{M}-\mathrm{H}]]^{-}$or protonated $[\mathrm{M}+\mathrm{H}]^{+}$molecular ions observed in the ESI-MS confirmed the molecular weights of the compounds.

\section{Antibacterial and Antifungal Activity}

The antibacterial and antifungal activity of compounds $\mathbf{2 a - j}$ was evaluated in vitro against the following strains: Pseudomonas aeruginosa ATCC 27853, Escherichia coli ATCC 25922, Klebsiella pneumoniae ATCC 4352, Proteus mirabilis ATCC 14153, Enterococcus faecalis ATCC 29212, Staphylococcus epidermidis ATCC 12228, Staphylococcus aureus ATCC 29213, Candida albicans ATCC 10231, Candida parapsilosis ATCC 22019 and Candida tropicalis ATCC 750. The compounds were prepared using twofold dilutions starting at $2500 \mu \mathrm{g} / \mathrm{mL}$. The lowest concentration of compound giving complete inhibition of visible growth was referred as the MIC (minimum inhibitory concentration).

As shown in Table 1, Staphylococcus epidermidis ATCC 12228, Staphylococcus aureus ATCC 29213 and Candida parapsilosis ATCC 22019 were the most sensitive strains to the tested hydrazone derivatives. All of the tested compounds, except for compound $\mathbf{2 h}$, showed weak antibacterial activity against Staphylococcus epidermidis ATCC 12228 and Staphylococcus aureus ATCC 29213 with MIC values of 312.5-1250 $\mu \mathrm{g} / \mathrm{mL}$. Compounds $\mathbf{2 a}$,

$\mathbf{2 b}, \mathbf{2 e}, \mathbf{2} \mathbf{f}$ and $\mathbf{2 i}$ further exhibited antifungal activity against Candida parapsilosis, showing complete inhibition at MIC val-

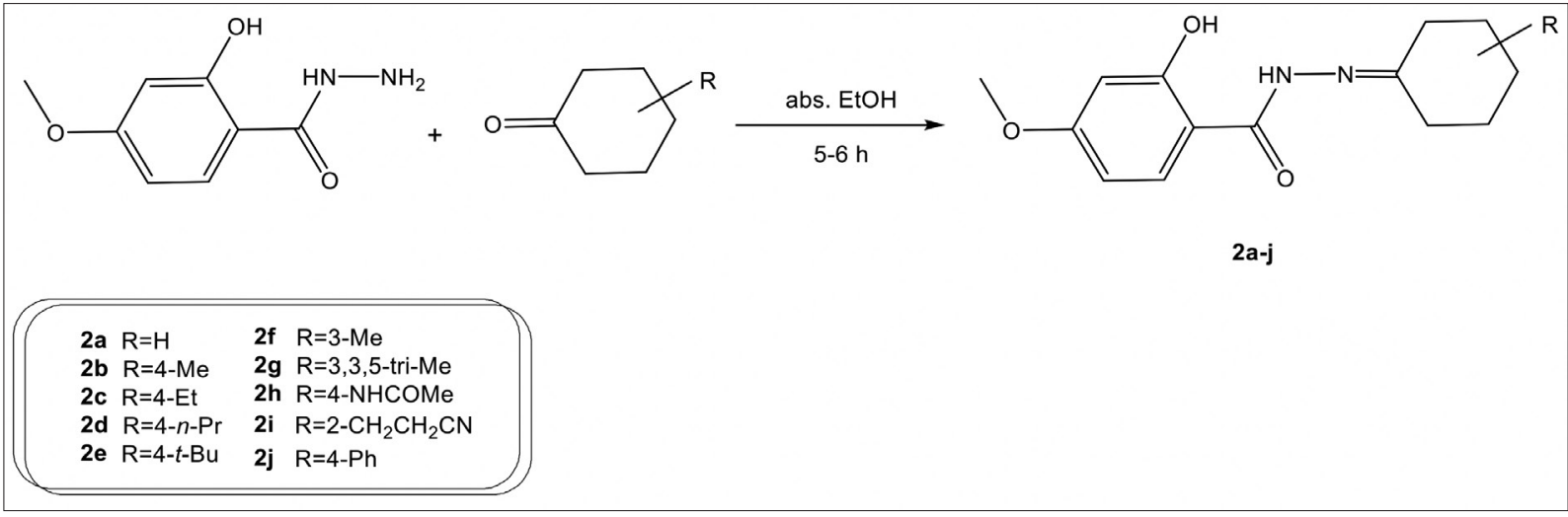

Scheme 1. Synthesis of $\mathbf{2 a - j}$. 
Table 1. Antimicrobial properties of compounds $2 a-j$ against selected bacteria and fungi

\begin{tabular}{|c|c|c|c|c|c|c|c|c|c|c|c|}
\hline \multirow[b]{2}{*}{ Microorganism } & \multicolumn{11}{|c|}{$\operatorname{MIC}(\mu \mathrm{g} / \mathrm{mL})^{a}$} \\
\hline & $2 a$ & $2 b$ & $2 c$ & $2 d$ & $2 e$ & $2 f$ & $2 g$ & $2 \mathrm{~h}$ & $2 \mathrm{i}$ & $2 \mathrm{j}$ & $\begin{array}{c}\text { Reference } \\
\text { antimicrobials }\end{array}$ \\
\hline P. aeruginosa ATCC 27853 & $-b$ & - & - & - & 625 & - & - & - & - & - & 2.4 (Ceftazidime) \\
\hline E. coli ATCC 25922 & - & - & - & - & - & - & - & - & - & -- & 4.9 (Cefuroxime-Na) \\
\hline K. pneumoniae ATCC 4352 & - & - & - & - & - & - & - & - & - & - & 4.9 (Cefuroxime-Na) \\
\hline P. mirabilis ATCC 14153 & - & - & - & - & - & - & - & - & - & - & 2.4 (Cefuroxime- $\mathrm{Na}$ ) \\
\hline E. faecalis ATCC 29212 & - & - & - & - & - & - & - & - & - & - & 128 (Amikacin) \\
\hline S. epidermidis ATCC 12228 & 625 & 1250 & 1250 & 1250 & 625 & 1250 & 1250 & - & 1250 & 625 & 9.8 (Cefuroxime) \\
\hline S. aureus ATCC 29213 & 625 & 1250 & 1250 & 1250 & 625 & 312.5 & 625 & - & 312.5 & 312.5 & 1.2 (Cefuroxime- $\mathrm{Na}$ ) \\
\hline C. albicans ATCC 10231 & - & - & - & - & - & - & - & - & - & - & 4.9 (Clotrimazole) \\
\hline C. parapsilosis ATCC 22019 & 312.5 & 312.5 & - & - & 625 & 625 & - & - & 312.5 & - & 0.5 (Amphotericin B) \\
\hline C. tropicalis ATCC 750 & - & - & - & - & - & - & - & - & - & - & 1 (Amphotericin B) \\
\hline
\end{tabular}

ues of 312.5 and $625 \mu \mathrm{g} / \mathrm{mL}$. Neither of the test compounds displayed antimicrobial activity below $312.5 \mu \mathrm{g} / \mathrm{mL}$.

Benzaldehyde phenylhydrazones incorporating an aromatic system linked to the hydrazide moiety have been extensively studied by different research groups (Kumar et al. 2010; Niazi et al. 2010; Tajudeen et al. 2013; Sapra et al. 2014; Backes et al. 2014). This type of compounds was found to be highly active against different bacteria and fungi species. Replacing the unsaturated aromatic ring with a saturated cyclohexane system seemed to have a negative effect on antimicrobial activity since compounds $\mathbf{2} \mathbf{a}$-j were found to be slightly active against the tested bacteria and fungi.

\section{Ethics Committee Approval: N/A.}

\section{Informed Consent: N/A.}

Peer-review: Externally peer-reviewed.

Author Contributions: Concept - G.C.Ü., E.M.K., G. C..; Design - G.C.Ü., E.M.K., G.Ç.; Supervision - G.Ç.; Resource - G. C.Ü., E.M.K., G.Ç.; Materials - G.C.Ü., E.M.K.; Data Collection and/or Processing - G.C.Ü., E.M.K.; Analysis and/or Interpretation - G.C.Ü., E.M.K.; Literature Search - G.C.Ü., E.M.K.; Writing - G.C.Ü., E.M.K.; Critical Reviews - G.Ç.

Conflict of Interest: The authors have no conflict of interest to declare.

Financial Disclosure: This work was supported in part by the Research Fund of Istanbul University (Project Number BYP-57695).

\section{REFERENCES}

- (2018) WHO-World Health Organization. Antibiotic resistance, https://www.who.int/news-room/fact-sheets/detail/antibioticresistance. Accessed 05.04.2019.

- Backes GL, Neumann DM, Jursic BS (2014). Synthesis and antifungal activity of substituted salicylaldehyde hydrazones, hydrazides and sulfohydrazides. Bioorg Med Chem 22: 4629-4636. [CrossRef]

- Clinical Laboratory Standards Institute (CLSI) document (1997). Approved standard-2nd edition M27-A2. Clinical and Laboratory Standards Institute. Wayne, PA, USA.
Clinical Laboratory Standards Institute (CLSI) document (2006). Approved standard-7th edition M7-A7. Clinical and Laboratory Standards Institute. Wayne, PA, USA.

Ge YQ, Li FR, Zhang YJ, Bi YS, Cao XQ, Duan GY, Wang JW, Liu ZL (2014). Synthesis, crystal structure, optical properties and antibacterial evaluation of novel imidazo[1,5-a]pyridine derivatives bearing a hydrazone moiety. Luminescence 29: 293-300. [CrossRef]

- Gürkök G, Altanlar N, Süzen S (2009). Investigation of antimicrobial activities of indole-3-aldehyde hydrazide/hydrazone derivatives. Chemotherapy 55: 15-19. [CrossRef]

- Kaplancıklı ZA, Yurttas L, Turan-Zitouni G, Özdemir A, Göğer G, Demirci F, Mohsen UA (2014). Synthesis and antimicrobial activity of new pyrimidine-hydrazones. Lett Drug Des Discov 11: 76-81. [CrossRef]

- Kumar D, Judge V, Narang R, Sangwan S, De Clercq E, Balzarini J, Narasimhan B (2010). Benzylidene/2-chlorobenzylidene hydrazides: Synthesis, antimicrobial activity, QSAR studies and antiviral evaluation. Eur J Med Chem 45: 2806-2816. [CrossRef]

- Laxminarayan R, Sridhar D, Blaser M, Wang M, Woolhouse M (2016). Achieving global targets for antimicrobial resistance. Science 353: 874-875. [CrossRef]

Luepke KH, Suda KJ, Boucher H, Russo RL, Bonney MW, Hunt TD, Mohr (2017). Past, present, and future of antibacterial economics: Increasing bacterial resistance, limited antibiotic pipeline and societal implications. Pharmacotherapy 37: 71-84. [CrossRef]

Moldovan C, Oniga O, Meda R, Tiperciuc B, Verite P, Pirnau A, Crisan O, Bojita M (2011). Synthesis and antimicrobial screening of novel 2, 3 or 4-[2-aryl-thiazol-ylmethoxy (oxo-ethoxy)]-benzaldehyde isonicotinoyl hydrazone analogs. Farmacia 59: 659-668.

- Morjan RY, Mkadmh AM, Beadham I, Elmanama AA, Mattar MR, Raftery J, Pritchard RG, Awadallah AM, Gardiner JM (2014). Antibacterial activities of novel nicotinic acid hydrazides and their conversion into N-acetyl-1,3,4-oxadiazoles. Bioorg Med Chem Lett 24: 5796-5800. [CrossRef]

Nastasa C, Tiperciuc B, Duma M, Benedec D, Oniga O (2015). New hydrazones bearing thiazole scaffold: Synthesis, characterization, antimicrobial, and antioxidant investigation. Molecules 20: 1732517338. [CrossRef]

Niazi S, Javali C, Paramesh M, Shivaraja S (2010). Study of influence of linkers and substitutions on antimicrobial activity of some Schiff bases. Int J Pharm Pharm Sci 2: 108-112. 
- Pieczonka AM, Strzelczyk A, Sadowska B, Mlostoń G, Stączek P (2013). Synthesis and evaluation of antimicrobial activity of hydrazones derived from 3-oxido-1H-imidazole-4-carbohydrazides. Eur J Med Chem 64: 389-395. [CrossRef]

- Popiolek L (2017). Hydrazide-hydrazones as potential antimicrobial agents: overview of the literature since 2010. Med Chem Res 26: 287-301. [CrossRef]

- Sapra A, Kumar P, Kakkar S, Narasimhan B (2014). Synthesis, antimicrobial evaluation and QSAR studies of $p$-hydroxy benzoic acid derivatives. Drug Res 64: 17-22. [CrossRef]

- Shlaes DM, Sahm D, Opiela C, Spellberg B (2013). The FDA reboot of antibiotic development. Antimicrob Agents Chemother 57: 4605-4607. [CrossRef]

- $\quad$ Silverstein RM, Webster FX, Kiemle DJ (2005). Spectrometric identification of organic compounds. 7th ed, John Wiley \& Sons, New York.

- Sridhar P, Alagumuthu M, Arumugam S, Reddy SR (2016). Synthesis of quinoline acetohydrazide-hydrazone derivatives evaluated as DNA gyrase inhibitors and potent antimicrobial agents. RSC Adv 6: 64460-64468. [CrossRef]

- Tacconelli E, Carrara E, Savoldi A, Harbarth S, Mendelson M, Monnet DL, Pulcini C, Kahlmeter G, Kluytmans J, Carmeli Y, Ouellette
M, Outterson K, Patel J, Cavaleri M, Cox EM, Houchens CR, Grayson ML, Hansen P, Singh N, Theuretzbacher U, Magrini N (2018). Discovery, research, and development of new antibiotics: the WHO priority list of antibiotic-resistant bacteria and tuberculosis. Lancet Infect Dis 18: 318-327. [CrossRef]

Tajudeen SS, Kannappan G (2013). Synthetic, structural and pharmacological studies on some isonicotinohydrazide and benzohydrazide analogues. J Pharm Res 7: 534-539. [CrossRef]

- Tatar E, Şenkardeş S, Sellitepe HE, Küçükgüzel ŞG, Karaoğlu ŞA, Bozdeveci A, De Clercq E, Pannecouque C, Hadda TB, Küçükgüzel I (2016). Synthesis and prediction of molecular properties and antimicrobial activity of some acylhydrazones derived from N-(arylsulfonyl)methionine. Turk J Chem 40: 510-534. [CrossRef]

- Vicini P, Zani F, Cozzini P, Doytchinova I (2002). Hydrazones of 1,2-benzisothiazole hydrazides: synthesis, antimicrobial activity and QSAR investigations. Eur J Med Chem 37: 553-564. [CrossRef]

- Xavier JJF, Krishnasamy K, Sankar C (2012). Synthesis and antibacterial, antifungal activities of some 2r,4c-diaryl-3-azabicyclo[3.3.1] nonan-9-one-4-aminobenzoyl hydrazones. Med Chem Res 21 345-350. [CrossRef] 